Article

\title{
Deep Insights into Gut Microbiota in Four Carnivorous Coral Reef Fishes from the South China Sea
}

\author{
Yu-Miao Gao ${ }^{1,2,+}$, Ke-Shu Zou ${ }^{1,2,+} \mathbb{0}$, Lei Zhou ${ }^{1,2}$, Xian-De Huang ${ }^{1,2}$, Yi-Yang $\mathrm{Li}^{1,2}$, \\ Xiang-Yang Gao ${ }^{3}$, Xiao Chen ${ }^{1,2, *}$ and Xiao-Yong Zhang 1,2,3,* \\ 1 Joint Laboratory of Guangdong Province and Hong Kong Region on Marine Bioresource Conservation and \\ Exploitation, College of Marine Sciences, South China Agricultural University, 483 Wushan Road, \\ Guangzhou 510642, China; gym_Seven@163.com (Y.-M.G.); zoukeshu@scau.edu.cn (K.-S.Z.); \\ zhoulei@scau.edu.cn (L.Z.); huangxd@scau.edu.cn (X.-D.H.); liyiyang0731@126.com (Y.-Y.L.) \\ 2 Guangdong Laboratory for Lingnan Modern Agriculture, Guangzhou 510642, China \\ 3 Guangdong Provincial Key Laboratory of Nutraceuticals and Functional Foods, College of Food Science, \\ South China Agricultural University, Guangzhou 510642, China; gaoxiangyang@scau.edu.cn \\ * Correspondence: chenxiao@scau.edu.cn (X.C.); zhangxiaoyong@scau.edu.cn (X.-Y.Z.); \\ Tel.: +86-20-8757-1321(X.-Y.Z.) \\ $+\quad$ Y.-M.G. and K.-S.Z. contributed equally to this work.
}

Received: 26 February 2020; Accepted: 16 March 2020; Published: 18 March 2020

check for updates

\begin{abstract}
Investigations of gut microbial diversity among fish to provide baseline data for wild marine fish, especially the carnivorous coral reef fishes of the South China Sea, are lacking. The present study investigated the gut microbiota of four carnivorous coral reef fishes, including Oxycheilinus unifasciatus, Cephalopholis urodeta, Lutjanus kasmira, and Gnathodentex aurolineatus, from the South China Sea for the first time using high-throughput Illumina sequencing. Proteobacteria, Firmicutes, and Bacteroidetes constituted $98 \%$ of the gut microbiota of the four fishes, and 20 of the gut microbial genera recovered in this study represent new reports from marine fishes. Comparative analysis indicated that the four fishes shared a similar microbial community, suggesting that diet type (carnivorous) might play a more important role in shaping the gut microbiota of coral reef fishes than the species of fish. Furthermore, the genera Psychrobacter, Escherichia-Shigella, and Vibrio constituted the core microbial community of the four fishes, accounting for $61-91 \%$ of the total sequences in each fish. The lack of the genus Epulopiscium in the four fishes was in sharp contrast to what has been found in coral reef fishes from the Red Sea, in which Epulopiscium was shown to be the most dominant gut microbial genus in seven herbivorous coral reef fishes. In addition, while unique gut microbial genera accounted for a small proportion $(8-13 \%)$ of the total sequences, many such genera were distributed in each coral reef fish species, including several genera (Endozoicomonas, Clostridium, and Staphylococcus) that are frequently found in marine fishes and 11 new reports of gut microbes in marine fishes. The present study expands our knowledge of the diversity and specificity of gut microbes associated with coral reef fishes.
\end{abstract}

Keywords: gut microbiota; carnivorous coral reef fishes; Illumina sequencing; the South China Sea

\section{Introduction}

Coral reefs, the most diverse and productive marine ecosystems on earth, are recognized as the rainforests of the sea [1]. It has been reported that coral reefs play a very important role in maintaining biodiversity and ecological balance of the oceans, although they occupy less than $0.1 \%$ of the world's ocean surface and provide complex and manifold marine habitats that support $25 \%$ of all marine 
species [2]. However, under the influence of climate change, disease outbreaks, overfishing, and even bad weather like hurricanes [3], the corals worldwide are undergoing great degradation, which also threatens the diversity of coral reef fishes [4]. As one of the most significant components of coral ecosystems, coral reef fishes can take a crucial role in protecting coral reef ecosystems from pathogen invasion. Most of the recent studies have mainly focused on investigating the diversity and distribution of coral reef fishes. So, despite the unparalleled species diversity and population density of coral reef fish reported in recent decades [5], very little is known about their intestinal tract microbiology [6].

The fish-associated symbiotic gut microbiota play a crucial role in nutritional provision and metabolic homeostasis. Nikouli et al. found that the gut microbial communities of farmed sea bream (Sparus aurata) fed different diets could influence the host's nutritional intake [7]. Another study reported that the intestinal microbiota of grass carp (Ctenopharyngodon idellus) fed with faba beans could significantly enhance the host's metabolic functions [8]. The gut microbiota can prevent the colonization of some infectious agents and maintain the host's immunity. Gupta et al. discovered that antibiotic-induced perturbations in Atlantic salmon could be regulated by the main gut microbial community of salmon [9]. Recent studies on the gut microbiota of fish have mainly focused on the manipulation of their diets and gut microbial communities to meet the needs of fish farming while trying to maintain host health and welfare.

However, investigations of gut microbial diversity among fish to provide baseline data from wild marine fish, especially from coral reef fishes, are still lacking. A few studies have revealed that the gut microbial community of the coral reef fish Kyphosus sydneyanus is markedly more abundant in larger fish than in smaller fish and that there is no specific microbial community distributed between the foregut and hindgut or between large fish and small fish [10]; the diet has a strong influence on the gut microflora, and there is a statistically significant correlation between the host phylogeny and gut microbiota in surgeon fishes [11]. Only a few studies on the gut microbiota of coral reef fishes have focused on herbivorous species and fermentation. The microbial community composition of carnivorous coral reef species has been relatively poorly investigated. Carnivorous fish exhibit a relatively high trophic status in the food chain and play a crucial role in maintaining the ecological balance of coral reef systems.

The goal of this study was to comprehensively investigate the diversity and structure of the microbial community associated with four carnivorous coral reef fishes, Oxycheilinus unifasciatus, Cephalopholis urodeta, Lutjanus kasmira, and Gnathodentex aurolineatus, from the South China Sea using high-throughput Illumina sequencing. Furthermore, we compared the gut microbial distribution in the four fish species and defined the core and unique microbial community, which provided data on the dominant gut microbiota in coral reef fishes from the South China Sea. Such information will expand our knowledge about the diversity and specificity of microbes associated with carnivorous fish species in coral reefs and help us understand the role of specific gut microbes. Based on the present study, we should achieve a better understanding of the relationship between the dietary and trophic structure of coral reef fish and their gut microbiota.

\section{Materials and Methods}

\subsection{Sample Collection}

Four species of coral reef fishes and 12 samples (Samples Ou1 Ou3; Cu1 Cu3; Lk1 Lk3, and Ga1 Ga3) were collected from Zhubi Reef $\left(10^{\circ} 55^{\prime} \mathrm{N}, 114^{\circ} 03^{\prime} \mathrm{E}\right)$ in the South China Sea in Aug 2017. All the coral reef fishes were identified by Dr. Xiao Chen (South China Agricultural University, Guangzhou, China) as belonging to the four species of Oxycheilinus unifasciatus (Samples Ou1 Ou3), Cephalopholis urodeta (Samples Cu1 Cu3), Lutjanus kasmira (Samples Lk1 Lk3), and Gnathodentex aurolineatus (Samples Ga1 Ga3) and were categorized as carnivorous coral reef fishes according to their diet in reference to descriptions from FishBase (https://www.fishbase.in/search.php, Copenhagen, Denmark). In addition, the phylogenetic tree based on the sequences of the cytochrome c oxidase 
subunit I (COI) genes of the four fish species downloaded from National Center for Biotechnology Information (NCBI, Bethesda, MD, USA) represented the evolutionary relationships between the four fishes. O. unifasciatus, G. aurolineatus, and L. kasmira were clustered onto one branch, which indicated that C. urodeta was less closely related to the other three fishes (Figure 1).

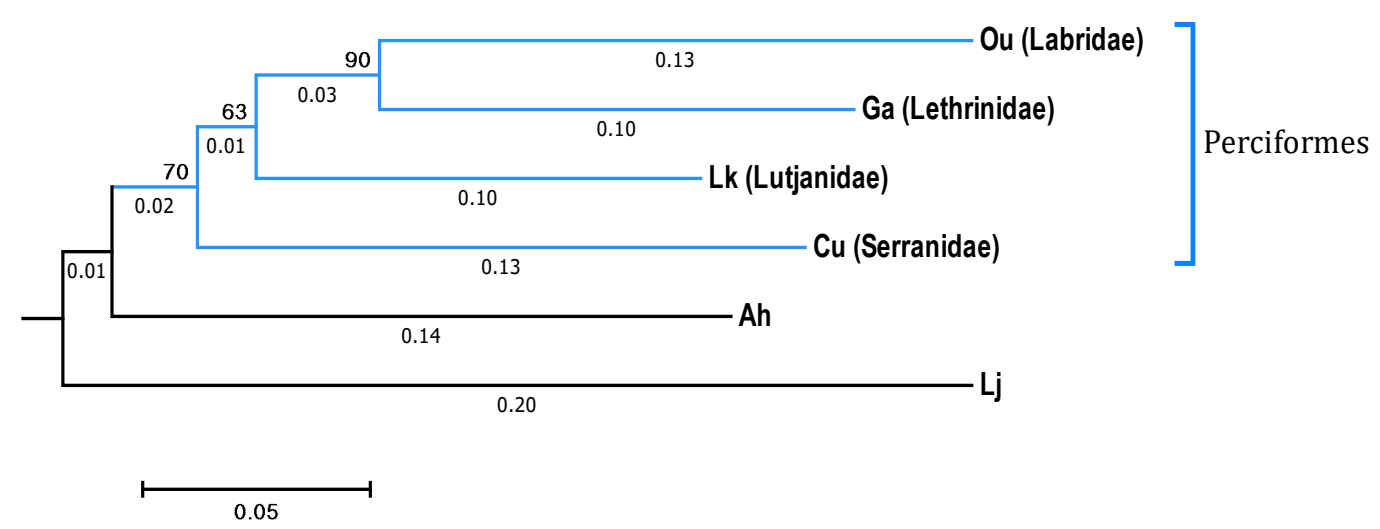

Figure 1. Neighbor-Joining phylogenetic tree of the cytochrome c oxidase subunit I (COI) gene of the four coral reef fishes from the South China Sea with the maximum composite likelihood and bootstrap method $(\mathrm{Q}=1000)$ was rooted by $\mathrm{Ah}$ (Acanthogobius hasta) and $\mathrm{Lj}$ (Lampetra japonicum) and constructed with mega 6.0. The clone sequences of the fish were downloaded from NCBI and the locus_tags were KC353468.1, NC_006131.1, MK567523.1, MK658630.1, FJ416614.1, MK566923.1. Ou: O. unifasciatus, Cu: C. urodeta, Lk: L. kasmira, Ga: G. aurolineatus.

These adult coral reef fishes were collected using hook and line and kept in sterile sea water. After anesthetization with $60 \mathrm{mg} / \mathrm{L}$ tricaine methanesulfonate, each fish was transported on ice to the laboratory as quickly as possible [11]. Under aseptic conditions, the external surface of the fish was cleaned with $75 \%$ ethanol to avoid contamination by the surface microbes on the fish. After opening the ventral surface using sterile scissors, the whole gut of each fish was aseptically removed and collected, and at least three replicates per sample were taken and assembled as one gut sample. A total of 12 gut samples from the four coral reef fishes was collected in frozen tubes and stored at $-80{ }^{\circ} \mathrm{C}$ until DNA extraction.

\subsection{Microbial DNA Extraction and $16 S$ rRNA Amplicon Sequencing}

The microbial DNA of each gut sample was extracted using the EZNA stool DNA Kit (Omega Biotek, Norcross, GA, USA) according to the manufacturer's protocols [1]. The V3-V4 region of the microbial 16S rRNA gene was amplified by PCR (initial denaturation at $95^{\circ} \mathrm{C}$ for $2 \mathrm{~min}$, followed by 27 cycles at $98{ }^{\circ} \mathrm{C}$ for $10 \mathrm{~s}, 62^{\circ} \mathrm{C}$ for $30 \mathrm{~s}$, and $68^{\circ} \mathrm{C}$ for $30 \mathrm{~s}$, with a final extension at $68{ }^{\circ} \mathrm{C}$ for $10 \mathrm{~min}$ ) using the forward primer CCTACGGRRBGCASCAGKVRVGAAG and the reverse primer GGACTACNVGGGTWTCTAATTCC, where the barcode was an eight-base sequence unique to each sample. PCR amplification was performed in triplicate in $50 \mu \mathrm{L}$ reactions containing $5 \mu \mathrm{L}$ of $2.5 \mathrm{mM}$ dNTPs, $5 \mu \mathrm{L}$ of $10 \times$ KOD buffer, $1.5 \mu \mathrm{L}$ of $5 \mu \mathrm{M}$ forward and reverse primers, $1 \mu \mathrm{L}$ of KOD polymerase, and approximately $100 \mathrm{ng}$ of template DNA.

The microbial 16S rRNA amplicon of each sample of the four fish species was extracted and purified with the AxyPrep DNA Gel Extraction Kit (Axygen Biosciences, Union City, CA, USA) following the manufacturer's operation guide. The purified microbial 16S rRNA amplicons were pooled in equimolar quantities and subjected to paired-end sequencing on the Illumina MiSeq platform (Illumina, San Diego, CA, USA) according to the manufacturer's protocols [12]. 


\subsection{Microbial Operational Taxonomic Units (OTUs) Cluster and Taxonomic Annotation}

The raw reads were merged as raw tags with FLAST ( $\mathrm{v}$ 1.2.11) according to an overlap of more than 10 bps and were further analyzed with Quantitative Insights Into Microbial Ecology (QIIME) software (Knight Lab, San Diego, CA, USA) (Version 1.9.1) [13,14], with processing under specific filtering conditions to improve the acuity of rare operational taxonomic unit (OTU) discrimination. The tags were searched against the reference database UCHIME (v 4.2) to remove chimeric sequences, and the effective tags were obtained. The further analyzed effective tags were clustered into operational taxonomic units (OTUs) at a 97\% similarity level using VSEARCH (1.9.6). In addition, the effective tag with the highest abundance was selected as a representative sequence for each cluster, which was assigned taxonomically using SILVA release 128 (Max Planck Institute for Marine Microbiology and Jacobs University, Bremen, Germany) [15].

\subsection{Alpha Diversity and Statistical Analysis}

Based on the OTU analysis results, a random sampling method was used to generate a rarefaction curve, and alpha diversity analysis was performed in R (R Core Team, Vienna, Austria) [16]. Chao 1 and the Shannon index were calculated and used throughout the study as indicators of richness and diversity, and other indices were included in tables for easy comparison with the existing literature [14]. Venn diagram analysis identifying core and unique OTUs was performed in R (R Core Team, Vienna, Austria) between groups [16]. The Ribosomal Database Project (RDP) classifier Bayesian algorithm was employed to carry out species taxonomy analysis on the representative sequences of OTUs and to quantify the community composition of each sample at different classification levels. The Unweighted Pair Group Method with Arithmetic Mean (UPGMA) phylogenetic tree was constructed by unweighted group averaging via hierarchical clustering [17].

\subsection{Nucleotide Sequence Accession Numbers}

All sequence data from the 12 coral reef fish samples were deposited in the Sequence Read Archive of the NCBI under accession number PRJNA603639.

\subsection{Ethics Statement}

All experiments were carried out in strict accordance with the recommendations of the Guide for the Care and Use of Laboratory Animals of the National Institutes of Health. The use of animals in this study was approved by Animal Ethics Committee at the South China Agricultural University, China (approval ID: 201004152, January 2010).

\section{Results}

\subsection{Sequence Overview}

After denoising and chimaera detection, a total of 677,841 microbial $16 \mathrm{~S}$ V4 sequences with a mean length of 458 bp were obtained and used for analysis, which were clustered into 191 OTUs with $97 \%$ sequence similarity. The average numbers of microbial OTUs detected in O. unifasciatus, C. urodeta, L. kasmira, and G. aurolineatus were 106, 125, 80, and 72, respectively (Table 1). Rarefaction curves for the four fishes constructed for the number of observed microbial sequences vs. OTUs showed a plateau ranging from 40 to 70 (Figure 2), indicating that the number of the obtained sequences could sufficiently represent the diversity of the gut microbes in each fish species. These results were supported by the comparison of the Shannon and Chao 1 indices and observed OTUs at $97 \%$ sequence similarity (Table 1). Good's nonparametric coverage estimate indicated that more than $99.9 \%$ of the diversity was recovered in each fish sample. 
Table 1. Comparison of microbial sequences, the estimated operational taxonomic units (OTUs) richness, Shannon's indexes of the microbial 16S V4 sequences for clustering at $97 \%$ sequences similarity from the sequencing analysis.

\begin{tabular}{cccccccc}
\hline Species of Coral Fishes & $\begin{array}{c}\text { Sequence } \\
\text { Read }\end{array}$ & $\begin{array}{c}\text { Avg Len } \\
\mathbf{( b \mathbf { p } )}\end{array}$ & OTUs & Chao1 & Shannon & Simpson & ACE \\
\hline Oxycheilinus unifasciatus & 53195 & 461 & 106 & 54 & 2.89 & 0.71 & 54.45 \\
\hline Cephalopholis urodeta & 69114 & 459 & 125 & 67 & 2.96 & 0.77 & 67.81 \\
\hline Lutjanus kasmira & 52213 & 458 & 80 & 53 & 2.55 & 0.65 & 53.84 \\
\hline Gnathodentex aurolineatus & 51425 & 454 & 72 & 46 & 2.63 & 0.65 & 47.75 \\
\hline
\end{tabular}

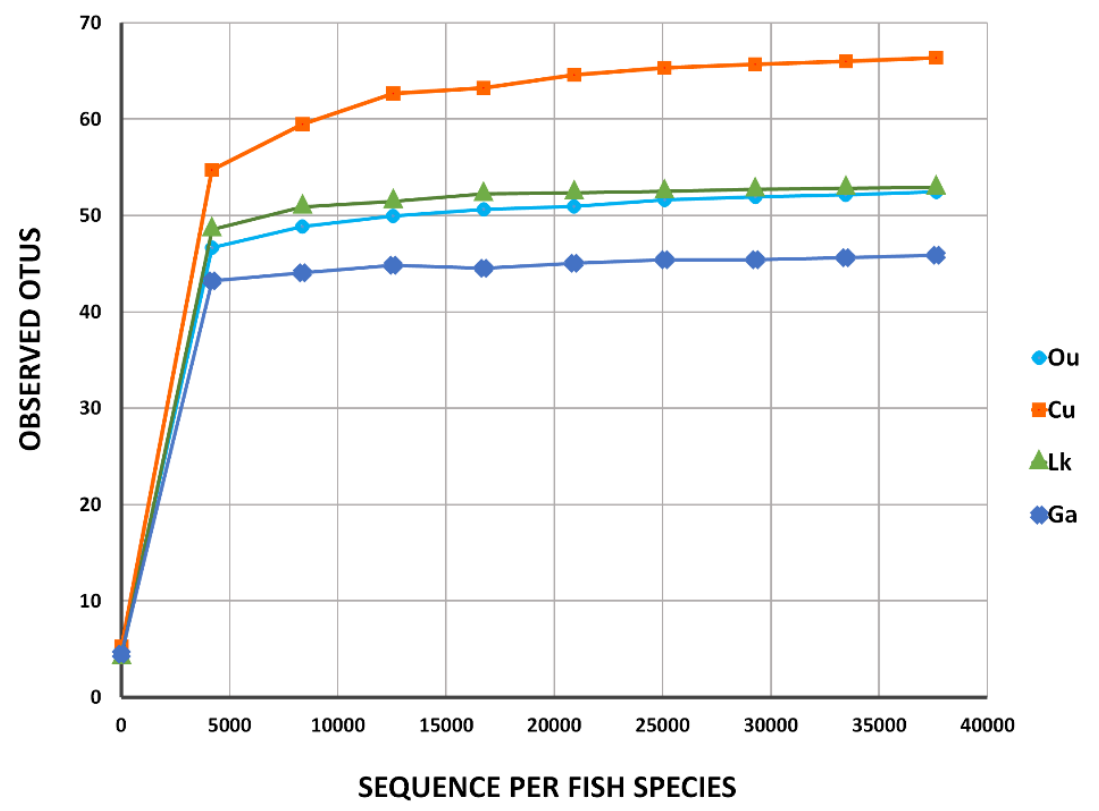

Figure 2. Rarefaction curves of four coral reef fishes from the South China Sea. Ou: O. unifasciatus, Cu: C. urodeta, Lk: L. kasmira, Ga: G. aurolineatus.

\subsection{Alpha Diversity}

The variation of the gut microbiota within each fish species was reflected in the alpha-diversity estimates obtained at $97 \%$ sequence identity (Table 1). C. urodeta exhibited the highest expected Chao 1 richness values and the highest variability between replicates $(n=3 ; 67 \pm 31)$. O. unifasciatus also presented high variability between replicates $(n=3 ; 54 \pm 30)$. In contrast, $G$. aurolineatus displayed the lowest expected mean richness values and the lowest variability in richness values between replicates $(n=3 ; 46 \pm 4)$. L. kasmira also showed low variability in the richness values between replicates $(n=3$; $53 \pm 9$ ). There was similar inter- and intraspecies variability in the Shannon index (Table 1). C. urodeta $(n=3 ; 2.97 \pm 0.40)$ exhibited the lowest variation with the highest average diversity. O. unifasciatus $(n=3 ; 2.89 \pm 1.06)$ presented the highest variation with comparably high diversity. The average diversity of L. kasmira $(n=3 ; 2.55 \pm 0.46)$ and G. aurolineatus $(n=3 ; 2.63 \pm 0.48)$ was low.

\subsection{Gut Microbial Composition}

The overall gut microbial composition of all fish samples at the phylum level displayed diverse assemblages of microbes consisting mainly of Proteobacteria (79\% of the total sequences), Firmicutes $(16 \%)$ and Bacteroidetes (3\%), with other phyla (Actinobacteria, Cyanobacteria, Saccharibacteria, Fusobacteria, Acidobacteria, Tenericutes, Deinococcus-Thermus and Verucomicrobia) accounting for less than $2 \%$ of the total sequences. 
When the taxonomic composition of each fish species at the phylum level was assessed, Proteobacteria was found to be the dominant phylum in the four coral fish species with other codominant phyla. C. urodeta, L. kasmira, and G. aurolineatus exhibited substantial proportions of Proteobacteria (82\%, $81 \%$, and $63 \%$, respectively) and Firmicutes $(16 \%, 17 \%$, and $32 \%$, respectively), while O. unifasciatus was dominated by Proteobacteria ( $89 \%$ ) and Bacteroidetes (9\%) (Figure 3). Little variation was observed in phylum-level taxonomic composition among replicates.

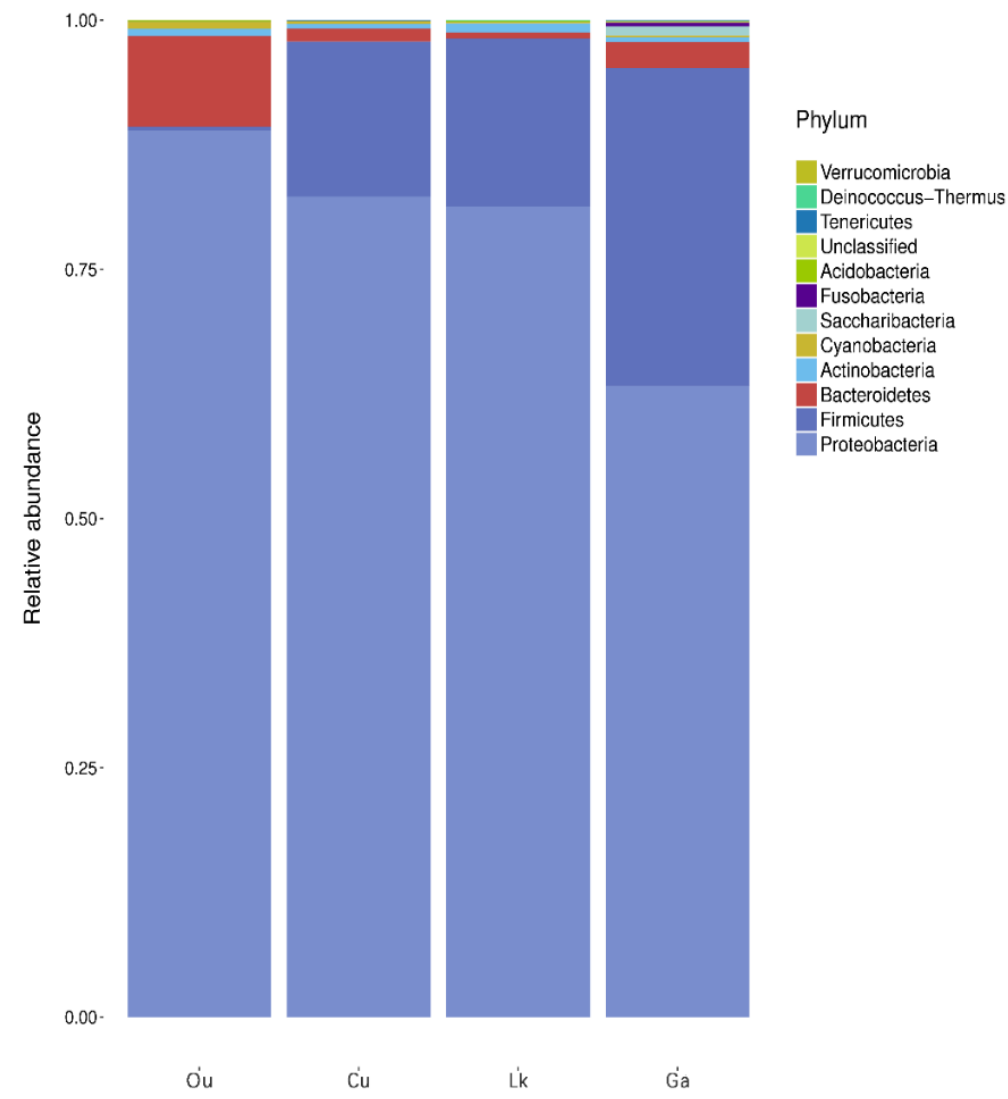

Figure 3. Taxonomic composition of gut microbes in four coral reef fishes at phylum level. Ou: $O$. unifasciatus, Cu: C. urodeta, Lk: L. kasmira, Ga: G. aurolineatus.

The taxonomic composition at the genus level was markedly more variable both between and within each fish species (Figure 4). Escherichia-Shigella was the dominant genus in O. unifasciatus and G. aurolineatus (43\% and 53\%, respectively). The most abundant genus in C. urodeta and L. kasmira was Psychrobacter (25\% and 50\%, respectively), while C. urodeta was characterized by the codominance of Escherichia-Shigella (15\%), an unclassified genus of Clostridiaceae (12\%), Vibrio (20\%), and Photobacterium $(14 \%)$. A phylogenetic tree was generated based on the 191 clustered OTUs and illustrated the top four phyla and top 30 genera in the four fish species (Figure 5). There was great intraspecies variability at the individual level, and the composition of the gut microbes in each fish species at the genus level may therefore be of doubt in some instances (Figure 4). For instance, the abundance of Vibrio in the three replicates of $C$. urodelus ranged from $0.01 \%$ to $46.45 \%$. An exception to this pattern was observed for G. aurolineatus, whose replicates were consistently dominated by Escherichia-Shigella (43-73\%). 


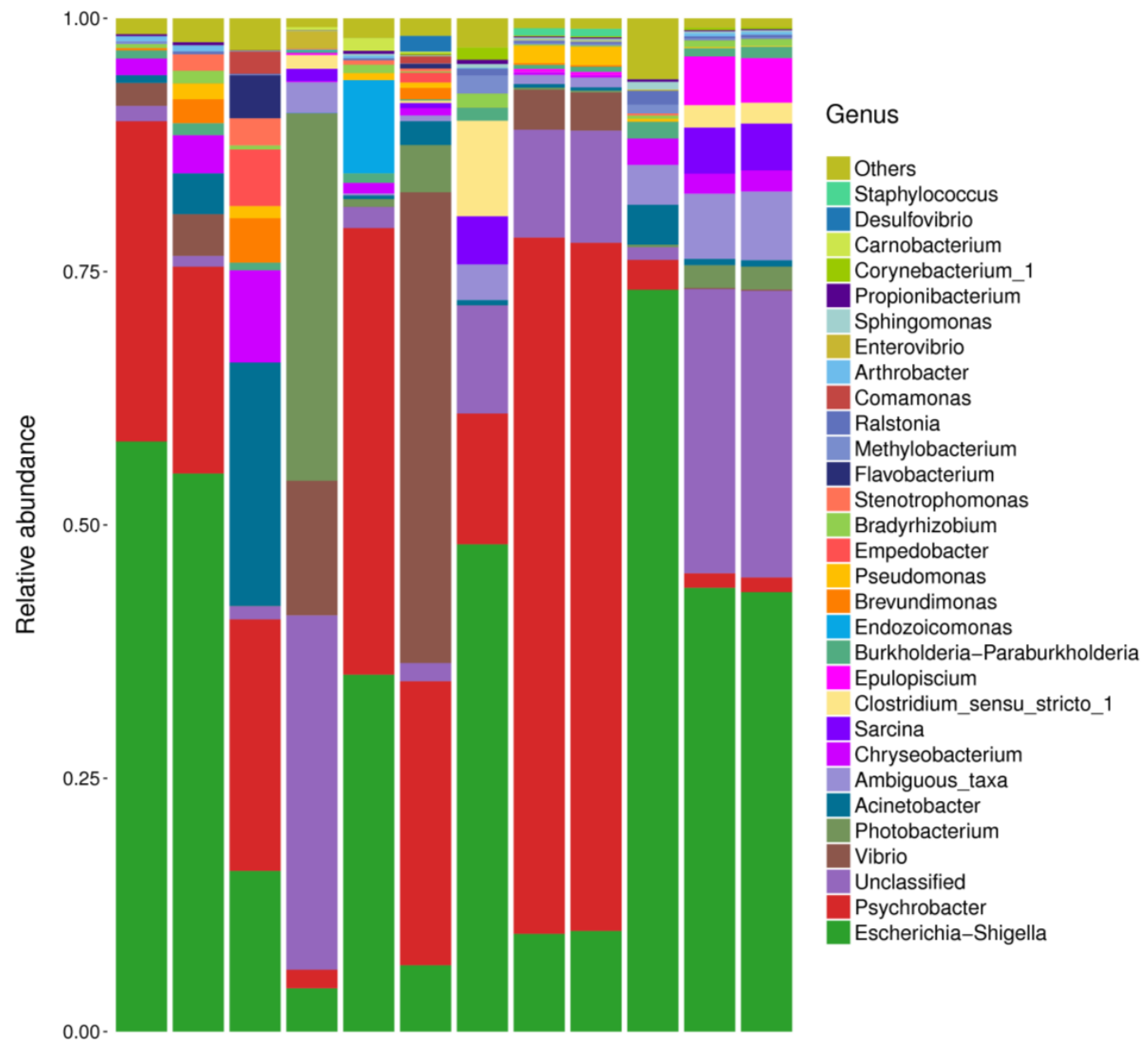

Ou1 Ou2 Oủ Cu1 Cu2 cu3 L'k1 L'k2 L'k3 Ga1 Ga2 Ga3

Figure 4. Interspecies gut microbial communities classified at the genus level. Ou: O. unifasciatus, Cu: C. urodeta, Lk: L. kasmira, Ga: G. aurolineatus. 


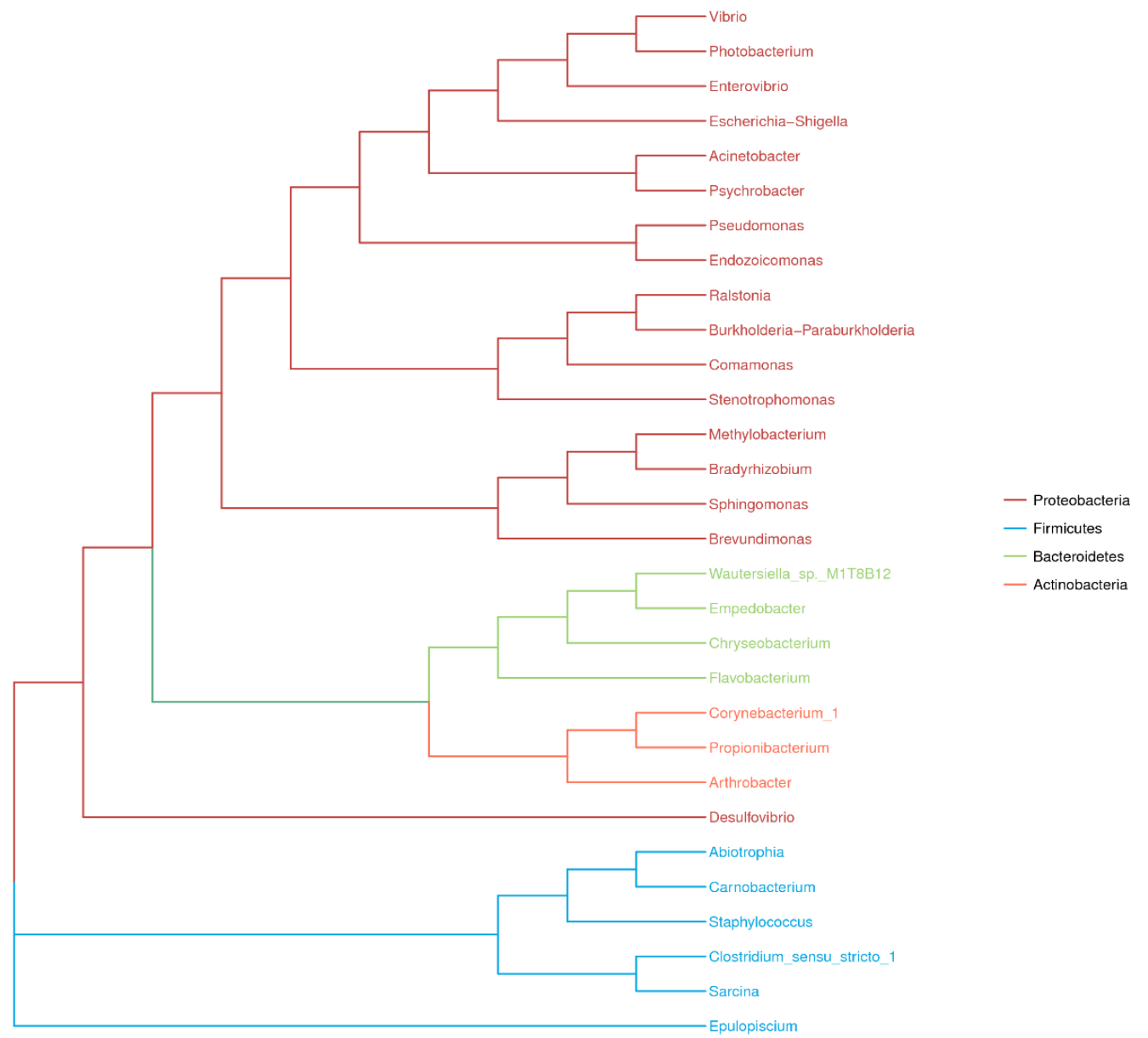

Figure 5. Phylogenic tree of the top 30 most abundant microbial OTUs recovered in the four fish species from the South China Sea revealed by high-throughput Illumina sequencing

\subsection{Core and Unique Gut Microbial Community in Four Coral Reef Fishes from the South China Sea}

The core gut microbes (the proportion of the microbial community shared between samples) were investigated for each species as a result of the observed high intraspecies variability. The proportions of the 24 shared OTUs among the replicates of each species were remarkably low, accounting for $8-13 \%$ of the total microbial OTUs (Figure 6). However, they accounted for a large fraction of the total sequences (68-84\%). The proportions of shared sequences in the replicates of O. unifasciatus, C. urodeta, L. kasmira, and G. aurolineatus were $64-86 \%, 77-89 \%, 72-91 \%$ and $61-87 \%$, respectively.

Many of the shared OTUs among the replicates for a species came from the abundant taxa in that species. For instance, the four fish species mainly shared Escherichia-Shigella, Psychrobacter, Vibrio and unclassified genera within phylum Clostridia. Other shared OTUs in the four fish species belonged to Acinetobacter, Burkholderia-Paraburkholderia, Bradyrhizobium, Propionibacterium, and an unclassified genus of Cyanobacteria. In addition, a small proportion of sequences belonging to Ralstonia, Arthrobacter, Propionibacterium, Tepidimonas, Methylobacterium, Pelomonas, Pseudomonas, and Acinetobacter were recovered from the four fishes. 


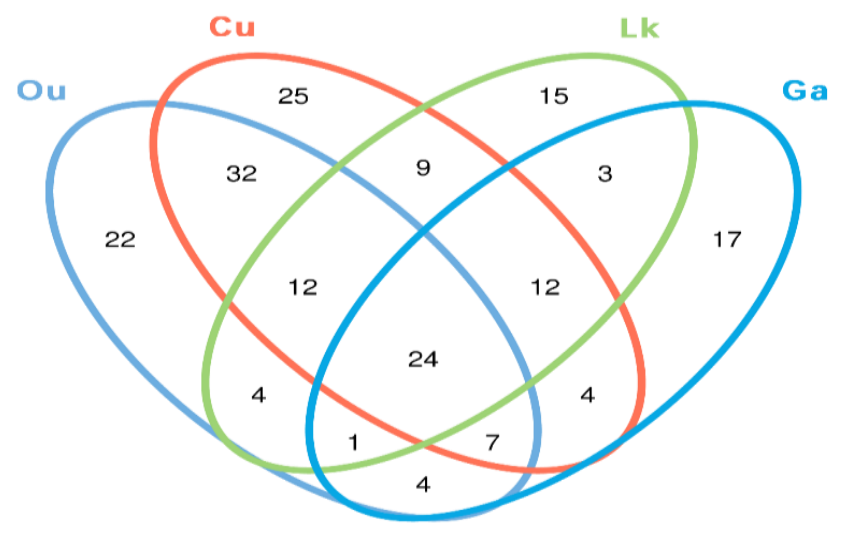

Figure 6. Venn diagram illustrates overlap of OTUs in gut microbiota among the four fish species. Ou: O. unifasciatus, Cu: C. urodeta, Lk: L. kasmira, Ga: G. aurolineatus.

The differences in the microbial communities of the four studied fishes were tested with via ANOSIM ( $R=0.352, p=0.018)$, showing no statistically significant differences in the microbial community among the four fish species (Figure 7). After further analysis, it was found that there were still some unique microbial OTUs recovered in different fish species (Figure 6 and Table 2). For example, Alkanindiges, Lysobacter, Olsenella, Paenibacillus, Porphyromonas, Rubritalea, Solibacillus, and Sphingopyxis were only recovered from the gut of O. unifasciatus, and 13 genera, including Carnobacterium, Desulfovibrio, Dolosigranulum, Endozoicomonas, Helcococcus, Massilia, Streptomyces, and five unclassified genera, were only detected in the gut of $C$. urodeta. In addition, the unique microbial community of L. kasmira consisted of Anaerotruncus, Clostridium, Deinococcus, Faecalibacterium, Hymenobacter, Macellibacteroides, Nesterenkonia, Nocardiopsis, Peptococcus, Rheinheimera, Roseomonas, and Staphylococcus, while six genera, Abiotrophia, Bosea, Gemella, Haliscomenobacter, Leptotrichia, and Veillonella, and an unclassified genus within Erysipelotrichaceae constituted the unique gut microbial community of G. aurolineatus.

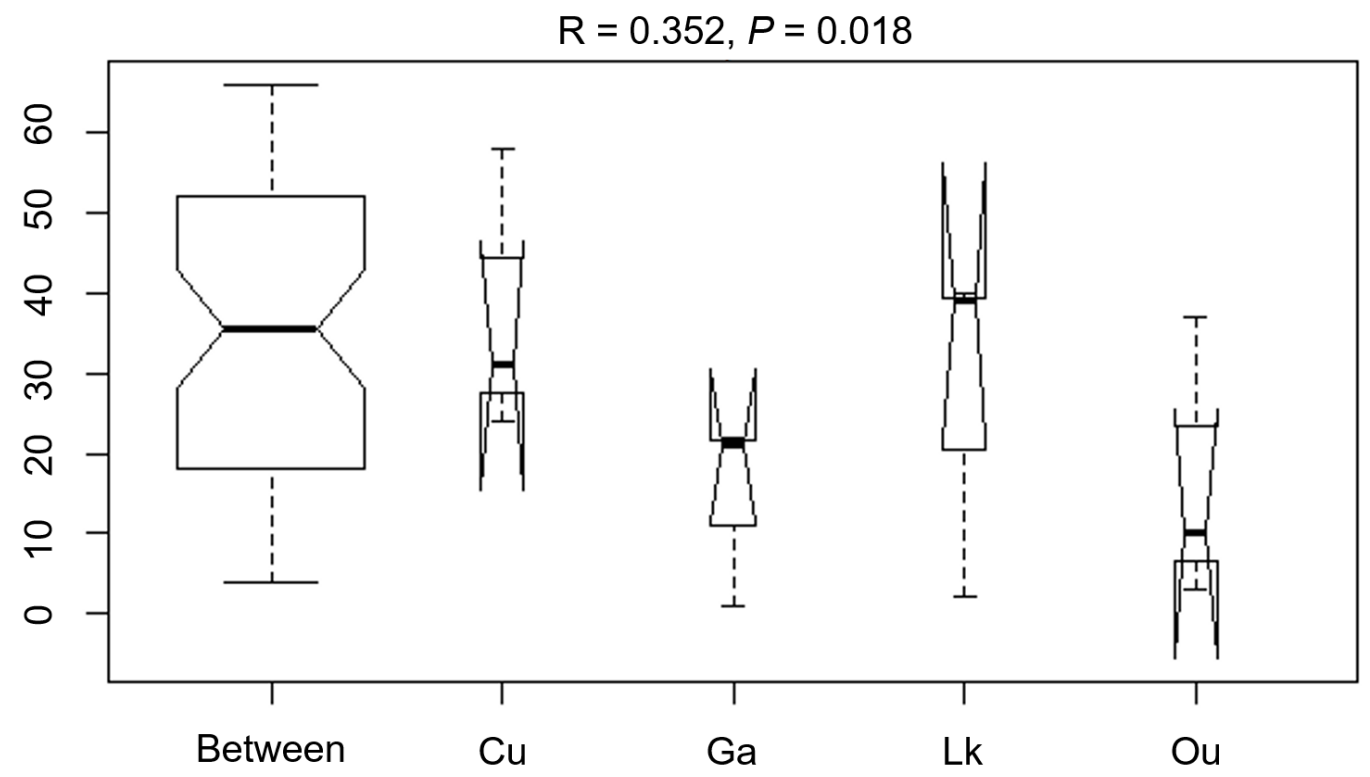

Figure 7. ANOSIM comparison (R statistics) of the four coral reef fishes from the South China Sea. Ou: O. unifasciatus, Cu: C. urodeta, Lk: L. kasmira, Ga: G. aurolineatus. 
Table 2. Unique gut microbial community at genus level in four different coral reef fishes from the South China Sea.

\begin{tabular}{cc}
\hline Fish Species & Unique Microbial Community \\
\hline Oxycheilinus unifasciatus & Alkanindiges, Lysobacter, Olsenella*, Paenibacillus, Porphyromonas, \\
Rubritalea, Solibacillus, Sphingopyxis
\end{tabular}

The genera marked by asterisk $\left(^{*}\right)$ are new reports for gut microbes of fishes.

\section{Discussion}

\subsection{New Insights into the Gut Microbial Communities of Coral Reef Fishes}

There is an apparent lack of research on the intestinal microflora of coral reef fishes, especially among carnivorous fish species from the South China Sea. This is the first investigation of the gut microbial communities of four carnivorous coral fish species (O. unifasciatus, C. urodeta, L. kasmira, and G. aurolineatus) from the South China Sea using high-throughput Illumina sequencing. In this study, an intriguing finding was that a total of 20 microbial genera, including g_11-24, Abiotrophia, Aquabacterium, BD1-7_clade, Bosea, Chryseomicrobium, Dolosigranulum, Erysipelotrichaceae_UCG-006, Family_XIII_AD3011_group, Haliscomenobacter, Leptotrichia, Leucobacter, Macellibacteroides, Massilia, Nocardiopsis, Olsenella, Peptococcus, Roseomonas, Ruminococcaceae_NK4A214_group, Ruminococcaceae_UCG-014, and Tepidimonas, were recovered for the first time from the guts of marine fishes (Table 3), which increased the number of gut microbial genera known from marine fishes. Among these 20 microbial genera, eight genera (indicated by asterisks in Table 3) are newly reported as gut microbes of fishes. The remaining 12 genera were previously reported in the gut microflora of freshwater fishes such as Ctenopharyngodon idellus [18], Fundulus heteroclitus [19], Oncorhynchus mykiss [20,21], Danio rerio [22], Carassius auratus [23], Carassius gibelio [24], and Coregonus baicalensis [25]. With the rapid development of modern molecular biotechnology, an increasing number of novel microbial communities and functions have recently been detected in the guts of fishes by using new techniques and methods [7-9]. Our results suggested that carnivorous coral reef fishes from the South China Sea harbor many previously unknown components of the gut microbial community. 
Table 3. Summary of new reports for gut microbiota in marine fishes.

\begin{tabular}{|c|c|c|c|c|c|c|}
\hline \multirow{2}{*}{ Microbial Genera } & \multicolumn{4}{|c|}{ Source Fishes } & \multirow{2}{*}{$\begin{array}{l}\text { Isolated } \\
\text { /Clone }\end{array}$} & \multirow{2}{*}{ Reference } \\
\hline & $\mathrm{Ou}$ & $\mathrm{Cu}$ & Lk & Ga & & \\
\hline g_11-24* & $\sqrt{ }$ & & & & clone & in this study \\
\hline Abiotrophia & & & & $\sqrt{ }$ & clone & in this study \\
\hline Aquabacterium & $\sqrt{ }$ & & $\sqrt{ }$ & & clone & in this study \\
\hline BD1-7_clade* & & $\sqrt{ }$ & & & clone & in this study \\
\hline Bosea & & & & $\sqrt{ }$ & clone & in this study \\
\hline Chryseomicrobium * & $\sqrt{ }$ & & $\sqrt{ }$ & $\sqrt{ }$ & clone & in this study \\
\hline Dolosigranulum * & & $\sqrt{ }$ & & & clone & in this study \\
\hline Erysipelotrichaceae_UCG-006* & & & & $\sqrt{ }$ & clone & in this study \\
\hline Family_XIII_AD3011_group * & & $\sqrt{ }$ & & & clone & in this study \\
\hline Haliscomenobacter & & & & $\sqrt{ }$ & clone & in this study \\
\hline Leptotrichia & & & & $\sqrt{ }$ & clone & in this study \\
\hline Macellibacteroides & & & $\sqrt{ }$ & & clone & in this study \\
\hline Massilia & & $\sqrt{ }$ & & & clone & in this study \\
\hline Nocardiopsis & & & $\sqrt{ }$ & & clone & in this study \\
\hline Olsenella & $\sqrt{ }$ & & & & clone & in this study \\
\hline Peptococcus & & & $\sqrt{ }$ & & clone & in this study \\
\hline Roseomonas & & & $\sqrt{ }$ & & clone & in this study \\
\hline Ruminococcaceae_NK4A214_group * & & $\sqrt{ }$ & & & clone & in this study \\
\hline Ruminococcaceae_UCG-014* & & $\sqrt{ }$ & & & clone & in this study \\
\hline Tepidimonas & $\sqrt{ }$ & $\sqrt{ }$ & $\sqrt{ }$ & $\sqrt{ }$ & clone & in this study \\
\hline
\end{tabular}

The genera marked by asterisk $\left({ }^{*}\right)$ are new reports for gut microbes of fishes, Ou: O. unifasciatus, $\mathrm{Cu}$ : C. urodeta,

Lk: L. kasmira, Ga: G. aurolineatus.

\subsection{Richness and Diversity Estimates of Gut Microbiota in Coral Reef Fishes}

The richness and diversity estimates of the gut microbiomes of the four coral reef fishes varied substantially (Chao1, 35-98; Shannon, 1.98-4.08) with an average Shannon's index of 2.76. These values were compared to estimates from other carnivorous coral reef fish species including Centropristis striata $(120 \pm 80.7 ; 3.1 \pm 2.76)$, Scomberomorus cavalla $(143 \pm 42.4 ; 1.79 \pm 0.05)$, Caranx hippos $(160 \pm 47.7$; $4.24 \pm 2.59)$, Sphyraena barracuda $(28.2 \pm 0.99 ; 0.69 \pm 0.87)$, Carcharhinus brevipinna $(107 \pm 102 ; 2.31 \pm 0.11)$ and Naso hexacanthus $178 \pm 18 ; 2.85 \pm 0.53$ ) [11,26]. However, conclusions obtained through different pipelines can be inconsistent, so the published diversity estimates are used for comparison as qualitative values [27]. The alpha diversity detected in this study suggested variation in richness between replicates in the guts of some coral reef fishes. This may be due to the complexity of the coral skeleton and reef structure in the coral ecosystem and the nutritional structure of the coral ecosystem.

\subsection{Comparison of Gut Microbial Community in Different Coral Reef Fish Species}

Several previous studies comparing the microbiota associated with coral reef fish species collected from the Gulf of Mexico and Southern Great Barrier Reef have shown a marked specificity between fish species and microbiota [28-31]. However, the results of ANOSIM conducted in this study indicated high similarity between the four fish species, probably because the four captured fishes mainly feed on small fishes and crustaceans, according to which the four fish species are classified as carnivores. This finding may demonstrate a certain correlation between the fish gut microflora and the diet of the fish. 
In a recent study, Miyake et al. revealed that many members of Firmicutes, especially in the Epulopiscium genus, constituted the most abundant microbial community in the guts of seven herbivorous coral reef fishes [11]. In contrast, Proteobacteria was the dominant phylum (63-89\%) in the four coral reef fish species included in the present study. C. urodeta, L. kasmira and G. aurolineatus exhibited codominance of Firmicutes (> 15\%). In O. unifasciatus, Firmicutes accounted for less than 1\%, but Bacteroidetes exhibited a relatively high abundance (9\%), including Chryseobacterium, Empedobacter and Flavobacterium. It has been reported that O. unifasciatus and C. urodeta feed mainly on fishes $(83 \%$ and $68 \%$, respectively) and crustaceans (14\% and 32\%, respectively); L. kasmira also feeds on fishes $(42 \%)$ and crustaceans (31\%) but consumes a variety of algae as well; and G. aurolineatus feeds mainly on crabs and gastropods, also consuming small fishes occasionally [32-34]. Among the four fish, $O$. unifasciatus and C. urodeta have a similar diet, with small fishes accounting for a large proportion of the diet, but there was a significant difference in the codominant phyla of the guts of these fish species, perhaps because $C$. urodeta is distantly related to O. unifasciatus phylogenetically (Figure 1). Our results suggested that the diet might play a more important role in shaping the gut microbiota of carnivorous coral reef fishes than the species of fish.

\subsection{Core and Unique Microbial Community in Different Coral Reef Fish Species}

A total of 24 OTUs affiliated with 15 genera were shared between the four coral reef fishes (core microbial community). Although the proportions of shared OTUs in each fish species were relatively low, accounting for 8-13\% of the total microbial OTUs, the shared sequences accounted for a large fraction of the total sequences (68-84\%) in each fish species. A similar result was found in a recent study by Miyake et al. [11]. Among the identified core gut microbiota, Psychrobacter, Escherichia-Shigella, and Vibrio were the most dominant genera in the four coral reef fishes. Psychrobacter is a widespread and evolutionarily successful microbial genus that can be recovered in high abundance from the guts of different marine fishes, such as Salvelinus alpinus [35] and Gadus morhua [36]. Psychrobacter has been reported to improve autochthonous microbial diversity along the GI tract and to improve feed utilization and innate immunity in Epinephelus coioides [37,38]. As conditional pathogens, Escherichia-Shigella and Vibrio are frequently detected in many healthy Actinopterygii fish species [39] and Atlantic salmon parr [40].

Although unique gut microbial genera accounted for a small proportion of the total sequences, there were many such genera in each coral reef fish species (Table 2). Interestingly, one to four of the genera among the unique gut microbiota in each fish species constituted new reports for marine fishes (Table 2). Most of the observed unique gut microbial genera can be frequently recovered from marine fishes. For example, Endozoicomonas spp. are often isolated from cardinalfish (Apogonidae) and damselfish (Pomacentridae) [41], and Clostridium spp. and Staphylococcus spp. are frequently recovered in pinfish (Lagodon rhomboids) [26,36].

\section{Conclusions}

In summary, the gut microbiota of four carnivorous coral reef fishes, O. unifasciatus, C. urodeta, L. kasmira, and G. aurolineatus, from the South China Sea were successfully characterized by high-throughput Illumina sequencing. Proteobacteria along with Firmicutes and Bacteroidetes constituted $98 \%$ of the gut microbiota of the four coral reef fishes from the South China Sea, and a total of 20 gut microbial genera recovered in this study represented new reports for marine fishes. ANOSIM indicated that the four carnivorous coral reef fishes harbored a similar microbial community, suggesting that diet type (carnivorous) might play a more important role in shaping the gut microbiota in coral reef fishes than the fish species. In addition, Psychrobacter, Escherichia-Shigella, and Vibrio constituted the core microbial community of the four carnivorous coral reef fishes. Although the unique gut microbial genera accounted for a small proportion of the total sequences, there were many such genera in each coral reef fish species, including several genera (Endozoicomonas, Clostridium and Staphylococcus) that are frequently found in marine fishes and 11 new reports of gut microbes in marine fishes. 
With the rapid development of modern molecular technology, an increasing number of gut microbiota in different marine organisms have been investigated using high-throughput sequencing methods. However, the relatively high analysis costs do not allow a large number of samples, and so it must be based on only a small number of samples, which may mean that the relatively limited data would have been different if supported by a higher sample base. Unfortunately, this is still the limit of this study. Therefore, more than three replicated samples should be included in the experiment in order to obtain more accurate data and results. Certainly, triplicate analysis on the same subject will be acceptable but not encouraged.

Author Contributions: X.-Y.Z. and X.C. conceived and designed the experiments; Y.-M.G. and K.-S.Z. performed the experiments; Y.-Y.L. and L.Z. conducted the data analysis; X.-D.H. and X.-Y.G. revised the manuscript; Y.-M.G. and X.-Y.Z. wrote the manuscript. All authors have read and agreed to the published version of the manuscript.

Funding: This study was financially supported by the National Natural Science Foundation of China (41666008 and3177100038), Program of Department of Ocean and Fisheries of Guangdong Province, China (A201701C03 and GDME2018C014) and Open Project Program of Guangdong Provincial Key Laboratory of Nutraceuticals and Functional Foods.

Conflicts of Interest: The authors declared that they have no competing interests.

\section{References}

1. Liu, Y.C.; Huang, R.M.; Bao, J.; Wu, K.Y.; Wu, H.Y.; Gao, X.Y.; Zhang, X.Y. The unexpected diversity of microbial communities associated with black corals revealed by high-throughput Illumina sequencing. FEMS Microbiol. Lett. 2018, 365, 167. [CrossRef]

2. Liao, B.; Xiao, B.; Li, Z. Coral Reef Ecosystem. In Symbiotic Microbiomes of Coral Reefs Sponges and Corals; Li, Z., Ed.; Springer: Dordrecht, The Netherlands, 2019; pp. 1-15.

3. Hughes, T.P. Catastrophes, Phase Shifts, and Large-Scale Degradation of a Caribbean Coral Reef. Science 1994, 265, 1547-1551. [CrossRef] [PubMed]

4. Jones, G.P.; McCormick, M.I.; Srinivasan, M.; Eagle, J.V. Coral decline threatens fish biodiversity in marine reserves. Proc. Natl. Acad. Sci. USA 2004, 101, 8251-8253. [CrossRef] [PubMed]

5. Roberts, C.M.; McClean, C.J.; Veron, J.E.N.; Hawkins, J.P.; Allen, G.R.; McAllister, D.E.; Mittermeier, C.G.; Schueler, F.W.; Spalding, M.; Wells, F.; et al. Marine biodiversity hotspots and conservation priorities for tropical reefs. Science 2002, 295, 1280-1284. [CrossRef] [PubMed]

6. Mountfort, D.O.; Campbell, J.; Clements, K.D. Hindgut fermentation in three species of marine herbivorous fish. Appl. Environ. Microbiol. 2002, 68, 1374-1380. [CrossRef]

7. Nikouli, E.; Meziti, A.; Antonopoulou, E.; Mente, E.; Kormas, K.A. Gut bacterial community in geographically distant populations of farmed sea bream (Sparus aurata) and sea bass (Dicentrarchus labrax). Microorganisims 2018, 6, 92. [CrossRef]

8. Zhou, L.; Lin, K.; Gan, L.; Sun, J.; Guo, C.; Liu, L.; Huang, X. Intestinal microbiota of grass carp fed feba beans: A comparative study. Microorganisims 2019, 7, 465. [CrossRef]

9. Gupta, S.; Fernandes, J.; Kiron, V. Antibiotic-induced perturbation are manifested in the dominant intestinal bacterial phyla of Atlantic salmon. Microorganisims 2019, 7, 233. [CrossRef]

10. Moran, D.; Turner, S.J.; Clements, K.D. Ontogenetic development of the gastrointestinal microbiota in the marine herbivorous fish Kyphosus sydneyanus. Microb. Ecol. 2005, 49, 590-597. [CrossRef]

11. Miyake, S.; Ngugi, D.K.; Stingl, U. Diet strongly influences the gut microbiota of surgeonfishes. Mol. Ecol. 2015, 24, 656-672. [CrossRef]

12. Chen, B.; Gao, L.; Pan, Q. Woody forages effect the intestinal bacteria diversity of golden pompano Trachinotus ovatus. AMB Express 2018, 8, 29. [CrossRef] [PubMed]

13. Magoc, T.; Salzberg, S.L. FLASH: Fast length adjustment of short reads to improve genome assemblies. Bioinformatics 2011, 27, 2957-2963. [CrossRef] [PubMed]

14. Caporaso, J.G.; Kuczynski, J.; Stombaugh, J.; Bittinger, K.; Bushman, F.D.; Costello, E.K.; Fierer, N.; Pena, A.G.; Goodrich, J.K.; Gordon, J.I.; et al. QIIME allows analysis of high-throughput community sequencing data. Nat. Methods 2010, 7, 335-336. [CrossRef] [PubMed] 
15. Bokulich, N.A.; Subramanian, S.; Faith, J.J.; Gevers, D.; Gordon, J.I.; Knight, R.; Mills, D.A.; Caporaso, J.G. Quality-filtering vastly improves diversity estimates from Illumina amplicon sequencing. Nat. Methods 2013, 10, 57-59. [CrossRef] [PubMed]

16. R Core Team. R: A Language and Environment for Statistical Computing; R Foundation for Statistical Computing: Vienna, Austria, 2014.

17. Cohen, F.A.C.; Pimentel, T.; Valenti, W.C.; Calado, R. First insights on the bacterial fingerprints of live seahorse skin mucus and its relevance for traceability. Aquaculture 2018, 492, 259-264. [CrossRef]

18. Ni, J.J.; Yan, Q.Y.; Yu, Y.H.; Wu, H.H.; Chen, F. Dispersal patterns of endogenous bacteria among grass carp (Ctenopharyngodon idellus) guts. Iran. J. Fish. Sci. 2017, 16, 605-618.

19. Givens, C.E. A Fish Tale: Comparison of the Gut Microbiome of 15 Fish Species and the Influence of Diet and Temperature on Its Composition. Ph.D. Thesis, University of Georgia, Athens, GA, USA, 2012; p. 232.

20. Nosi, M.; Fadaak, S.; Danish-Daniel, M.; Iehata, S. Assessment of gut microbiota in different developmental stages of Malaysian Mahseer (Tor tambroides). Aquac. Res. 2018, 49, 2977-2987. [CrossRef]

21. Ricaud, K.; Rey, M.; Plagnes-Juan, E.; Larroquet, L.; Even, M.; Quillet, E.; Skiba-Cassy, S.; Panserat, S. Composition of Intestinal Microbiota in Two Lines of Rainbow Trout (Oncorhynchus mykiss) Divergently Selected for Muscle Fat Content. Open Microbiol. J. 2018, 12, 308-320. [CrossRef]

22. Basili, D.; Lutfi, E.; Falcinelli, S.; Balbuena-Pecino, S.; Navarro, I.; Bertolucci, C.; Capilla, E.; Carnevali, O. Photoperiod Manipulation Affects Transcriptional Profile of Genes Related to Lipid Metabolism and Apoptosis in Zebrafish (Danio rerio) Larvae: Potential Roles of Gut Microbiota. Microb. Ecol. 2019. [CrossRef]

23. Li, J.; Ni, J.; Li, J.; Wang, C.; Li, X.; Wu, S.; Zhang, T.; Yu, Y.; Yan, Q. Comparative study on gastrointestinal microbiota of eight fish species with different feeding habits. J. Appl. Microbiol. 2014, 117, 1750-1760. [CrossRef]

24. Cui, H.; Wang, Z.; Liu, J.; Wang, Y.; Wang, Z.; Fu, J.; Wan, Z.; Li, R.; Li, Q.; Fitton, H.J.; et al. Effects of a highly purified fucoidan from Undaria pinnatifida on growth performance and intestine health status of gibel carp Carassius auratus gibelio. Aquac. Nutr. 2020, 26, 47-59. [CrossRef]

25. Belkova, N.L.; Sidorova, T.V.; Glyzina, O.Y.; Yakchnenko, V.M.; Sapozhnikova, Y.P.; Bukin, Y.S.; Baturina, O.A.; Sukhanova, L.V. Gut microbiome of juvenile coregonid fishes: Comparison of sympatric species and their F1 hybrids. Fundam. Appl. Limnol. 2017, 189, 279-290. [CrossRef]

26. Givens, C.; Ransom, B.; Bano, N.; Hollibaugh, J. Comparison of the gut microbiomes of 12 bony fish and 3 shark species. Mar. Ecol. Prog. Ser. 2015, 518, 209-223. [CrossRef]

27. Siegwald, L.; Caboche, S.; Even, G.; Viscogliosi, E.; Audebert, C.; Chabé, M. The impact of bioinformatics pipelines on microbiota studies: Does the analytical "microscope" affect the biological interpretation? Microorganisms 2019, 7, 393. [CrossRef] [PubMed]

28. Egerton, S.; Culloty, S.; Whooley, J.; Stanton, C.; Ross, R.P. The Gut Microbiota of Marine Fish. Front. Microbiol. 2018, 9, 873. [CrossRef] [PubMed]

29. Smriga, S.; Sandin, S.A.; Azam, F. Abundance, diversity, and activity of microbial assemblages associated with coral reef fish guts and feces. FEMS Microbiol. Ecol. 2010, 73, 31-42. [CrossRef] [PubMed]

30. Larsen, A.; Tao, Z.; Bullard, S.A.; Arias, C.R. Diversity of the skin microbiota of fishes: Evidence for host species specificity. FEMS Microbiol. Ecol. 2013, 85, 483-494. [CrossRef] [PubMed]

31. Nielsen, S.; Walburn, J.W.; Vergés, A.; Thomas, T.; Egan, S. Microbiome patterns across the gastrointestinal tract of the rabbitfish Siganus fuscescens. Peer J. 2017, 5, e3317. [CrossRef]

32. Westneat, M.W. Transmission of force and velocity in the feeding mechanisms of labrid fishes (Teleostei, Perciformes). Zoomorphology 1994, 114, 103-118. [CrossRef]

33. Randall, J.E.; Brock, V.E. Observations on the ecology of Epinepheline and Lutjanid fishes of the society islands, with emphasis on food habits. Trans. Am. Fish. Soc. 1960, 89, 9-16. [CrossRef]

34. Mablouké, C.; Kolasinski, J.; Potier, M.; Cuvillier, A.; Potin, G.; Bigot, L.; Frouin, P.; Jaquemet, S. Feeding habits and food partitioning between three commercial fish associated with artificial reefs in a tropical coastal environment. Afr. J. Mar. Sci. 2013, 35, 323-334. [CrossRef]

35. Ransom, B.L. Intestinal Microbial Community Composition of Six Actinopterygii Fish Species in the Southeastern United States; University of Georgia: Athens, GA, USA, 2008.

36. Dehler, G.E.; Secombes, C.J.; Martin, S.A.M. Environmental and physiological factors shape the gut microbiota of Atlantic salmon parr (Salmo salar L.). Aquaculture 2017, 467, 149-157. [CrossRef] [PubMed] 
37. Yang, H.L.; Sun, Y.; Ma, R.L.; Li, J.S.; Huang, K.P. Probiotic Psychrobacter sp. improved the autochthonous microbial diversity along the gastrointestinal tract of grouper Epinephelus coioides. J. Aquac. Res. Dev. 2011, s1, 001.

38. Sun, Y.Z.; Yang, H.L.; Ma, R.L.; Zhang, C.X.; Lin, W.Y. Effect of dietary administration of Psychrobacter sp. on the growth, feed utilization, digestive enzymes and immune responses of grouper Epinephelus coioides. Aquac. Nutr. 2011, 17, 733-740. [CrossRef]

39. Ringo, E.; Sperstad, S.; Myklebust, R.; Mayhew, T.M.; Olsen, R.E. The effect of dietary insulin on aerobic bacteria associated with hindgut of Arctic charr (Salvelinus alpinus L.). Aquac. Res. 2006, 37, 891-897. [CrossRef]

40. Ringo, E.; Sperstad, S.; Myklebust, R.; Refstie, S.; Krogdahl, A. Characterisation of the microbiota associated with intestine of Atlantic cod (Gadus morhua L.): The effect of fish meal, standard soybeanmeal and a bioprocessed soybean meal. Aquaculture 2006, 261, 829-841. [CrossRef]

41. Parris, D.J.; Brooker, R.M.; Morgan, M.A.; Dixson, D.L.; Stewart, F.J. Whole gut microbiome composition of amselfish and cardinalfish before and after reef settlement. Peer J. 2016, 4, e2412. [CrossRef]

(C) 2020 by the authors. Licensee MDPI, Basel, Switzerland. This article is an open access article distributed under the terms and conditions of the Creative Commons Attribution (CC BY) license (http://creativecommons.org/licenses/by/4.0/). 